\title{
Impaired emotion processing and a reduction in trust in patients with somatic symptom disorder
}

\author{
Maja Erkic ${ }^{1}$ Josef Bailer ${ }^{1} \quad$ Sabrina C. Fenske ${ }^{1} \quad$ Stephanie N. L. Schmidt ${ }^{1} \quad$ Jörg Trojan $^{2}$ \\ Annette Schröder ${ }^{3}$ Peter Kirsch $^{1}$ (D) Daniela Mier ${ }^{1}$ (D)
}

${ }^{1}$ Department of Clinical Psychology, Central Institute of Mental Health, Medical Faculty Mannheim, University Heidelberg, Mannheim, Germany

${ }^{2}$ Department of Psychology, University of Koblenz-Landau, Landau, Germany

${ }^{3}$ Department of Clinical Psychology and Psychotherapy, University of Koblenz-Landau, Landau, Germany

Correspondence

Daniela Mier, Department of Clinical

Psychology, Central Institute of Mental Health,

Medical Faculty Mannheim, University

Heidelberg, J5, 68159 Mannheim, Germany.

Email: daniela.mier@zi-mannheim.de

\begin{abstract}
There is accumulating evidence for deficits in the perception and regulation of one's own emotions, as well as the recognition of others' emotions in somatic symptom disorder (SSD). However, investigations of SSD focusing on specific aspects of emotion processing and how these might interact are missing. We included 35 patients with SSD and 35 healthy controls who completed questionnaires on the perception and regulation of their own emotions, as well as experimental investigations of emotion recognition and trust. In line with previous studies, our results show that SSD patients in comparison to healthy controls have difficulties in the identification and description of own feelings $\left(\eta_{p}{ }^{2}=.381\right.$ and $\left.\eta_{p}{ }^{2}=.315\right)$. Furthermore, we found that patients apply less cognitive reappraisal $\left(\eta_{p}{ }^{2}=.185\right)$ but tend to use more expressive suppression $\left(\eta_{p}{ }^{2}=.047\right)$. In contrast to previous studies, we found SSD patients to perform superior in emotion recognition, in particular for anger $(d=0.40)$. In addition, patients with SSD invested less in a trust game $(d=0.73$ ). These results point to a higher sensitivity for negative emotions and less trust in others. Further, these findings suggest a dissociation between the ability to recognize one's own emotions versus others' emotions in SSD. Future interventions targeting emotion processing in SSD might focus on the identification of one's own emotions, prior to the training of emotion regulation.
\end{abstract}

KEYWORDS

alexithymia, emotion recognition, emotion regulation, somatic symptom disorder, trust

\section{INTRODUCTION}

Although the key features of somatic symptom disorders (SSD) are prominent somatic symptoms, patients with unexplained somatic symptoms also experience a decrease in quality of life, including high frequency of absence rates at work, limitations in daily activities, and a lower general health perception (Hanssen, Lucassen, Hilderink, Naarding, \& Voshaar, 2016; Leonidou, Panayiotou, Bati, \& Karekla, 2016; Rask, Ørnbøl, Rosendal, \& Fink, 2017; Spitzer et al., 1995). In addition, SSD is associated with high levels of negative affective states and alexithymia (Waller \& Scheidt, 2004). At present, cognitive behavioural therapy (CBT) is the method of choice in the treatment of SSD (Gottschalk \& Rief, 2012). However, CBT has been shown to be less effective in SSD than in other mental disorders, raising the question which specific characteristics of SSD patients are not completely addressed by CBT (Gottschalk, Bleichhardt, Kleinstäuber, Berking, \& Rief, 2015). Therefore, recent approaches in psychotherapy have started addressing the improvement of emotion processing abilities in somatoform patients (Gottschalk et al., 2015; Kleinstäuber, Gottschalk, Berking, Rau, \& Rief, 2016). To support the further development of emotion focused therapies for SSD, it is necessary to get a deeper understanding of emotion processing impairments in SSD.

Medically unexplained physical symptoms are a common problem in primary care patients (Rief \& Martin, 2014). About $16 \%$ to $31 \%$ of consultations in general practice are based on somatic symptoms without adequate medical explanation (Sauer \& Eich, 2007). About $26 \%$ to $35 \%$ of these patients fulfil the diagnostic criteria for a Diagnostic and Statistical Manual of Mental Disorders (DSM)-IV somatoform disorder (SFD; Haller, Cramer, Lauche, \& Dobos, 2015; Saß, Wittchen, \& Zaudig, 1996). According to the German Health Interview and Examination Survey, SFD has a 12-month prevalence of $11 \%$ and a lifetime prevalence of $16.2 \%$ (Wittchen \& Jacobi, 2001). The reorganized DSM-5 category somatic symptom and related disorders, which replaces SFD, is defined by positive symptoms, that is, distressing somatic complaints accompanied by abnormal thoughts, feelings, and behaviours, emphasizing the role of psychological factors. Further, in comparison to 
DSM-IV, the presence of a medical disorder is no longer an exclusion criterion. For easier reference, we will present earlier studies on patients with medially unexplained symptoms under the umbrella of SSD, albeit is has to be kept in mind that these studies relied on former classifications of SDD patients with slightly divergent diagnostic criteria.

Early characterizations of patients with somatic symptoms report impairments in affect perception and expression (MacLean, 1949). Based on the descriptions of patients with reduced abilities to experience and describe their emotions, the concept of alexithymia was introduced (Nemiah \& Sifneos, 1970). The authors used this term to describe a group of patients who are unable to understand their own feelings and to link the resulting bodily sensations to affective arousal, which makes them prone to develop physical complaints, and to attribute bodily sensations as signs of disease. Further, such a decreased emotional awareness has been associated with difficulties to differentiate bodily sensations and to distinguish between affective arousal and physical symptoms (Burton, Weller, \& Sharpe, 2009; Subic-Wrana, Beutel, Knebel, \& Lane, 2010; Taylor, 2000). Recent studies have shown that patients with medically unexplained somatic symptoms fail to link their emotions to experienced physical complaints and misinterpret physiological aspects of affective arousal as bodily symptoms (Burton et al., 2009; Subic-Wrana et al., 2010). Moreover, the disability to understand and express one's own feelings was linked to immune alterations, a negative impact on health, and has been assumed to represent a risk factor for psychosomatic and stress-related illness (de Timary, Roy, Luminet, Fillee, \& Mikolajczak, 2008; Lumley, Stettner, \& Wehmer, 1996; Uher, 2010). Regarding symptom reports, especially self-reported difficulties in identifying feelings, as measured with the Toronto Alexithymia Scale (TAS; Bagby, Parker, \& Taylor, 1994), show strong correlations with the number of somatic symptoms (Bailer, Witthöft, Erkic, \& Mier, 2017; De Gucht \& Heiser, 2003; Deary, Scott, $\&$ Wilson, 1997).

Furthermore, individuals with high levels of alexithymia seem to use maladaptive emotion regulation strategies enhancing physiological aspects of emotional experience, suggesting that patients with SSD might also rely on dysfunctional emotion regulation styles (Laloyaux, Fantini, Lemaire, Luminet, \& Laroi, 2015; Swart, Kortekaas, \& Aleman, 2009). Traue (1998) postulates that somatization is promoted by the suppression of negative feelings, which is in accordance with studies that have shown that suppression enhances physiological aspects of emotional experience (Alexander, 1950; Pennebaker \& Seagal, 1999). Moreover, the usage of suppression as emotion regulation strategy was shown to be associated with higher levels of alexithymia (Kessler, Kammerer, Hoffmann, \& Traue, 2010; Nemiah \& Sifneos, 1970). Bucci (1997a) argued that patients with SSD exhibit impaired cognitive regulation of affective arousal and therefore experience isolated somatic sensations without intact connection to the corresponding cognition, which leads to a persistent physical activation and promotes psychosomatic disorders. Taylor, Bagby, and Parker (1997) also assumed that this patient group is impaired in using cognitive mechanisms in order to identify and regulate emotions, resulting in enhanced focus on physiological sensations that accompany affective arousal without linking them to a specific emotion. This might further enhance the bodily perceptions and lead to a misinterpretation of bodily sensations as

\section{Key Practitioner Message:}

- Patients with somatic symptom disorders (SSD) have difficulties in identifying and describing their own feelings, while they have an intact perception of others' emotions.

- Patients with SSD seem to be especially sensitive towards negative emotions and to have less trust in others.

- Patients with SSD apply less cognitive reappraisal in order to regulate emotions and tend to use more expressive suppression.

- A lack of trust may contribute to the frequent medical consultations in SSD.

- Psychotherapies in SSD should comprise a focus on improving the understanding and regulation of the patients' emotions.

indicators of illness. However until now, the number of studies applying questionnaires or experimental designs to investigate the way of coping with negative emotions in somatoform patients is still limited.

Interestingly, it was shown that especially in those SSD patients with higher alexithymia scores, not only the ability to recognize one's own emotions seemed to be impaired but also the ability to adequately identify others' emotions (Pedrosa Gil et al., 2009). Congruently, the recognition of others' emotions is becoming a field of interest in SSD as well, because it is an important skill regarding interpersonal interactions and interpersonal emotion regulation (Zaki \& Williams, 2013). It might therefore have an impact on social functioning in general, as well as on the course of disease (Schönenberg et al., 2014; Waller \& Scheidt, 2006). Indeed, it has been shown that SSD patients perform worse than individuals without somatic symptoms on emotion recognition tasks (Beck, Breuss, Kumnig, \& Schussler, 2013). Buhlmann, Etcoff, and Wilhelm (2006) investigated self-referential emotion recognition in patients with body dysmorphic disorder and found patients having a negative bias. Beck et al. (2013) also showed impaired emotion recognition abilities in SSD patients and stressed the importance of therapeutic interventions aiming at the improvement of emotion recognition and regulation. Schönenberg et al. (2014) found intact facial affect perception in patients with persistent somatoform pain disorder. However, by using videos of social scenes, they observed impaired mentalizing abilities in the patient group. The authors further argued that due to this deficit, SSD patients might tend to misinterpret social signals that can cause distress in social interactions. Considering that SSD patients might tend to label affective expressions of others as negative in a self-referential context (Buhlmann et al., 2006), interactions with physicians during medical examinations and social interactions in general are predisposed to evoke negative feelings, resulting in less trust. This might contribute to the high number of medical consultations with frequently changing physicians, as often observed in patients with SSD (de Zwaan \& Müller, 2006). 
Taken together, patients with SSD seem to be affected in various facets of emotion processing and social cognition, ranging from deficits in the perception and regulation of their own emotions to the recognition of others' emotions and intentions. We assume to find the deficits in emotion processing that were reported for patients diagnosed with former versions of the DSM also in patients with SSD.

We expect that patients with SSD use significantly more expressive suppression than healthy controls. Further, we hypothesize that patients with SSD are impaired in identifying and describing their own feelings and show deficits in recognizing feelings of others. With regard to social interactions, we expect patients with SSD to show less trust than healthy controls.

Further, we were interested in investigating the interplay of the emotion processing deficits and how they contribute to the medically unexplained bodily symptoms in SSD. We assumed that alexithymia and emotion regulation deficits both contribute to impaired recognition of others' emotions in SSD. Finally, we were interested whether alexithymia mediates the influence of emotion regulation skills on medically unexplained bodily symptoms.

\section{METHODS}

\subsection{Sample}

In total, 35 patients with SSD and 35 healthy controls participated in the study. The group of SSD patients was recruited in the context of an ongoing multicenter therapy study, which was designed to compare the efficacy of conventional CBT with CBT enriched with strategies addressing emotion processing and regulation (ENCERT; Kleinstäuber et al., 2016).

We asked those patients of the ENCERT study enrolled for a treatment in the outpatient clinics of the University of Koblenz-Landau or the Central Institute for Mental Health Mannheim (CIMH) to take part in our study. In sum, 14 patients from Landau and 21 patients from the $\mathrm{CIMH}$ agreed to participate. Patients were on average 42 years old (standard deviation $[S D]=13$ ), and $57.1 \%$ were female. Thirty-five healthy control subjects, who were matched by age, gender, and years of education, were included in the study. Table 1 shows a detailed description of the characteristics of both groups.

\subsection{Study design and procedures}

If patients had given written consent during the diagnostic phase of the ENCERT study, we contacted them, explained the study objective and procedures, and arranged an appointment. Healthy controls were recruited with advertisements on the website of the $\mathrm{CIMH}$ and information flyers on billboards in Mannheim. Interested participants were included if they did not fulfil the criteria of any mental disorder, currently or in the past, as assessed by a telephone version of the Structured Clinical Interview for DSM-IV Axis I and II (Wittchen, Zaudig, \& Fydrich, 1997). The procedure was approved by the local ethics board of the Medical Faculty Mannheim of the University of Heidelberg. Before being enrolled in the study, participants gave written informed consent.

Diagnoses in the patient group were assessed by applying the Structured Clinical Interview for DSM-IV, too. The inclusion criteria for the patients were based on the diagnoses of somatization disorder

TABLE 1 Sample characteristics and symptom measures $(M \pm S D)$

\begin{tabular}{|c|c|c|c|}
\hline & $\begin{array}{l}1 \\
\text { SSD } \\
(n=35)\end{array}$ & $\begin{array}{l}2 \\
\mathrm{HC} \\
(n=35)\end{array}$ & $\begin{array}{l}t \text { test } / \chi^{2} \text { test } \\
t / \chi^{2} \text { value }\end{array}$ \\
\hline Age in years $(M \pm S D)$ & $42.4 \pm 12.9$ & $41.1 \pm 12.1$ & $t(68)=.430$ \\
\hline Gender: female (\%) & 57.1 & 57.1 & $\chi^{2}(1, N=70)<.001$ \\
\hline School education ( $\geq 12$ years; \%) & 45.7 & 51.4 & $t(68)=.431$ \\
\hline \multicolumn{4}{|l|}{ Somatoform symptoms } \\
\hline PHQ-15 (M $\pm S D)$ & $13.5 \pm 5.1$ & $1.7 \pm 1.9$ & $t(68)=12.837, p<.001$ \\
\hline SOMS number of symptoms $(M \pm S D)$ & $12.4 \pm 8.8$ & $3.4 \pm 4.8$ & $t(68)=5.303, p<.001$ \\
\hline SOMS intensity of symptoms $(M \pm S D)$ & $0.8 \pm 0.59$ & $0.1 \pm 0.12$ & $t(68)=7.165, p<.001$ \\
\hline \multicolumn{4}{|l|}{ Depressive symptoms } \\
\hline $\mathrm{BDI}(M \pm S D)$ & $21.6 \pm 11$ & $2.5 \pm 4.3$ & $t(68)=9.576, p<.001$ \\
\hline \multicolumn{4}{|l|}{ Current DSM-IV diagnosis n (\%) } \\
\hline Somatization disorder ${ }^{a}$ & $6(17.1)$ & 0 & \\
\hline Undifferentiated somatoform disorder ${ }^{a}$ & $24(68.6)$ & 0 & \\
\hline Pain disorder ${ }^{a}$ & $5(14.3)$ & 0 & \\
\hline Any co-morbid mental disorder ${ }^{a}$ & $19(54.3)$ & 0 & \\
\hline Current depression $^{\mathrm{a}}$ & $12(34.3)$ & 0 & \\
\hline Current anxiety disorder ${ }^{a}$ & $8(22.9)$ & 0 & \\
\hline Any personality disorder ${ }^{a}$ & $5(14.3)$ & 0 & \\
\hline
\end{tabular}

Note. SSD = somatic symptom disorder group; HC = healthy control group; PHQ-15 = Patient Health Questionnaire; SOMS = Screening for Somatoform Disorders; $\mathrm{BDI}=$ Beck Depression Inventory; $\mathrm{M}=$ mean; SD = standard deviation; DSM = Diagnostic and Statistical Manual of Mental Disorders.

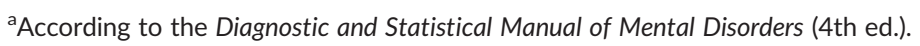


and undifferentiated somatoform disorder according to DSM-IV as well as on the DSM-5 diagnosis of SSD. According to Kleinstäuber and colleagues (2016), the DSM-5 criteria for SSD are better suited to detect patients in need for treatment, but additional criteria should be applied to ensure symptom severity. Therefore, the DSM- 5 criteria were adapted to the presence of at least three distressing physical symptoms with duration of at least 6 months. Following the criteria for SSD, at least one of three psychological criteria had to be fulfilled as well: disproportionate thoughts, persistently high level of anxiety, or excessive time and energy devoted in regard to symptoms or health concerns (see DSM-5, American Psychiatric Association, 2013).

In addition, as empirically proven cut-off criteria (Mewes et al., 2009), a self-reported Pain Disability Index (PDI) score $\geq 4$ (Dillmann, Nilges, Saile, \& Gerbershagen, 1994) and a Patient Health Questionnaire (PHQ)-15 total score $\geq 5$ were applied to confirm symptom severity (Kroenke, Spitzer, \& Williams, 2002). Patients with alcohol, drug, or substance addiction, psychosis, or acquired brain injuries were excluded. Other co-morbid mental disorders were allowed, unless the co-morbid disorder was considered to be the major problem. Further, patients were excluded from the study if symptoms could be fully explained by a medical disease, which had to be verified by a medical report.

\subsection{Self-report questionnaires}

Difficulties in the processing of one's own emotions were measured with the TAS-20, containing the subscales difficulties identifying feelings (DIF, 7 items), difficulties describing feelings (DDF, 5 items), and externally oriented thinking (EOT, 8 items) as well as a total score (TAS-20; Bagby et al., 1994). Reliability estimates in the current study were high for the two subscales DIF and DDF (Cronbach's $\alpha=.87$ and .80 ) but lower for the subscale EOT $(a=.52$ ). Deficits in the ability to cope with emotions were assessed with the Emotion Regulation Questionnaire, which distinguishes the subscales cognitive reappraisal (6 items) and expressive suppression (4 items; Abler \& Kessler, 2009). In our sample, Cronbach's a was .85 for cognitive reappraisal and .75 for expressive suppression. Additional questionnaires were used to assess: The number and intensity of somatic symptoms during the past 7 days (Screening for Somatoform Disorders-7T; Rief \& Hiller, 2008), symptom severity (PHQ-15; Kroenke et al., 2002), the degree to which different aspects of life are disrupted by pain (PDI; Dillmann et al., 1994), as well as depressive symptoms (Beck Depression Inventory
Revised-II; Hautzinger, Keller, \& Kühner, 2006). These instruments had good reliabilities in our sample, with a coefficients for Screening for Somatoform Disorders-7T (47 items) .94 for the number and .97 for the intensity of symptoms, PHQ-15 (15 items) .89, PDI (7 items) .94 , and Beck Depression Inventory (21 items) .96. Means and SDs of the questionnaire scores are shown in Table 1 and Figures 1 and 2.

\section{$2.4 \quad$ Experimental tasks}

In the emotion recognition task (Fenske et al., 2015), face stimuli with angry, neutral, or happy facial expressions were presented following positive, neutral, or negative pictures of scenes. This task was selected, because it can be assumed to combine emotion recognition with emotion regulation (Fenske et al., 2015). Participants were instructed to pay attention to both pictures, but to indicate the face valence only, by pressing the appropriate key on a standard computer keyboard as fast as possible. The pictures of scenes were taken from the International Affective Picture System (IAPS; Lang, Bradley, \& Cuthbert, 1999) and combined either positive valence and high arousal (e.g., sport activities), negative valence and high arousal (e.g., crime scenes), or neutral valence and low arousal (e.g., daily situations). Pictures of negative and positive scenes were matched for arousal, which was significantly higher than in neutral pictures. Positive and negative pictures were also matched to have a similar difference in valence compared to pictures of neutral scenes (Fenske et al., 2015). The facial stimuli were taken from the "NimStim set of facial expressions" (Tottenham et al., 2009) and consisted of 5 male and 5 female faces. To avoid ceiling effects, the facial stimuli were morphed according to a $60 \%$ emotional and $40 \%$ neutral ratio, as provided by Matzke, Herpertz, Berger, Fleischer, and Domes (2014). IAPS pictures were presented for $3 \mathrm{~s}$, and the immediately following facial expressions were shown until the response key was pressed but for $3 \mathrm{~s}$ at most (Figure 3 ). The facial expression was followed by a masking stimulus consisting of a pattern of random black and white pixels, which was presented for $500 \mathrm{~ms}$. The task included 90 trials consisting of 10 trials for each of the 9 combinations of each IAPS category (positive, neutral, and negative) with each face category (happy, neutral, and angry) and took about $11 \mathrm{~min}$.

In the adapted Trust Game (Franzen et al., 2011; Lis, Schönwetter, Mier, Gallhofer, \& Kirsch, 2011), participants were first shown the portrait pictures of four coplayers (i.e., trustees) on a computer screen and were asked to rate how likeable they seem to them ("sympathy rating" from "1 = not at all likeable" to "9 = very likeable"). Participants were in

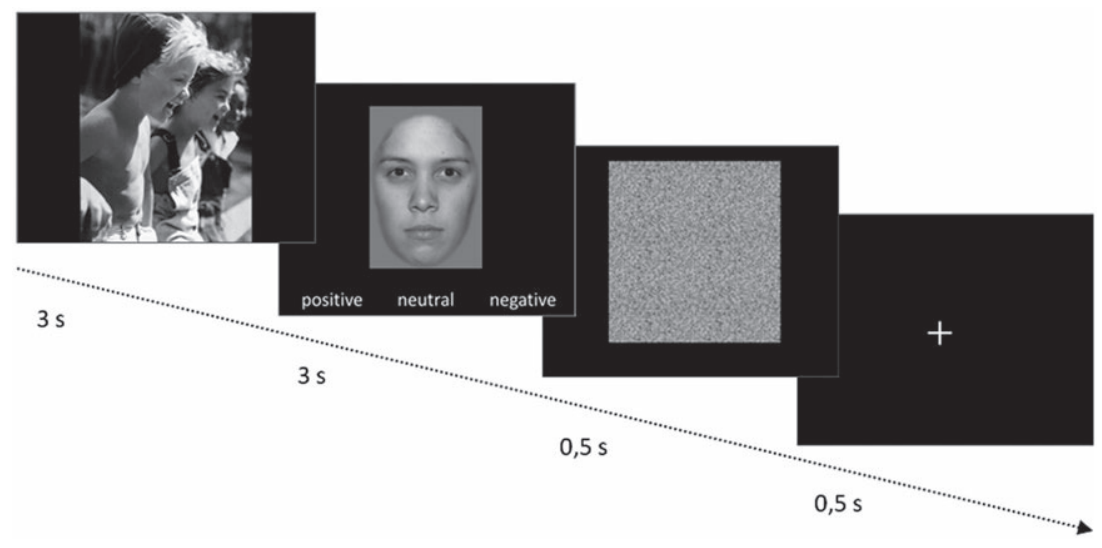

FIGURE 1 Emotion recognition task. Pictures of scenes are presented first, followed by pictures of happy, neutral, or angry facial expressions, a masking stimulus and a fixation cross. Numbers represent the duration of the stimulus presentation. Example with a positive scene and a neutral facial expression 


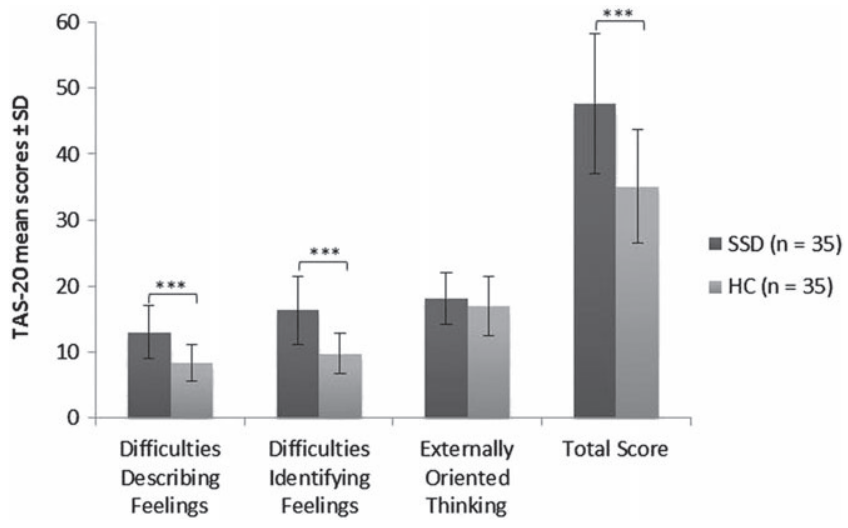

FIGURE 2 Toronto Alexithymia Scale (TAS)-20 mean scores and standard deviations $(S D)$ of patients (somatic symptom disorder [SSD]) and healthy controls (HC) for the three subscales of the TAS-20 and the total score. ${ }^{* * *} p<.001$

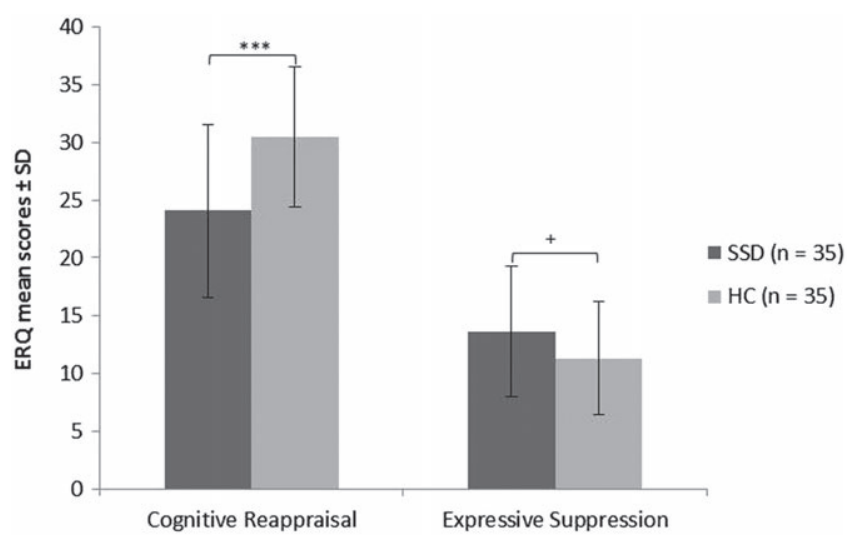

FIGURE 3 Emotion Regulation Questionnaire (ERQ) mean scores and standard deviations (SD) of patients (somatic symptom disorder [SSD]) and healthy controls $(\mathrm{HC})$ for the two emotion regulation strategies cognitive reappraisal and expressive suppression. ${ }^{* * *} p<.001 .^{+} p<.1$

the role of the investor. They were given 90 monetary units at the beginning of each round. The picture of a trustee was presented, and the participants had to decide how many of their 90 monetary units (10 units at least) they would like to transfer to the trustee. The chosen amount could be logged in by moving the mouse cursor to the according value presented on the screen. The transferred monetary units were tripled and credited to the trustee, who in return could transfer an amount of monetary units back to the participant, finishing one round. Return values of the trustees were enquired previously in an independent experimental setting. Therefore, no cover story was needed in the present experiment. They played four rounds, and in each round, the participants gained the sum of monetary units they kept plus the monetary units they reobtained from the trustee. Afterwards, one of the four rounds was selected randomly, and the gained monetary units were converted into money, which the participants actually received at the end of the experiment. Finally, the participants were asked to rate their coplayers once more. The questions were to rate again how likeable the trustees seemed to them after having played together, and in addition, how fair the trustees acted during the trust game ("fairness rating" from " 1 = not at all fair" to "9 = very fair"). Giving instructions, performing the task and the ratings took about $5 \mathrm{~min}$.
Due to delays in preparing the trust game and the necessity to start the project in the realms of the ENCERT study, the trust game was implemented later on. Thus, for the analyses, data from only 18 patients and 31 healthy control participants is available. In this subgroup, $55.6 \%$ of the participating patients and $54.5 \%$ of the healthy controls were female. The average age and average years of education of both groups were comparable to the total sample (participants of both groups were on average 42 years old $(S D=13)$, about $44.4 \%$ of patients and $45.2 \%$ of controls had a school education of at least 12 years).

Both experiments were implemented in the Presentation software, Version 17 (Neurobehavioral Systems, Albany, CA, USA). It should be noted that participants also performed a short task on somatosensory discrimination (grating orienting task), which is beyond the scope of this paper and will be reported elsewhere.

\subsection{Statistical analyses}

Main effects and condition by group interactions were analysed with repeated measures analysis of variances and multivariate analysis of variances. In case of violations of sphericity, Greenhouse-Geisser correction was applied. Bonferroni adjusted post-hoc analyses, as well as differences between groups in the trust game (in terms of sympathyand fairness-ratings as well as average investment rate) were analysed by two-sample $t$ tests and Mann-Whitney $U$ tests, as the data were not normally distributed. Effect sizes are reported as partial $\eta^{2}$ values $\left(\eta^{2} \geq .01, \eta^{2} \geq .06\right.$, and $\eta^{2} \geq .14$ are defined as small, medium, and large effects), Cohen's $d$ ( $d \geq 0.2, d \geq 0.5$, and $d \geq 0.8$ are defined as small, medium, and large effects) and $r(r \geq .1$ defined as small, $r \geq .3$ as medium, and $r \geq .5$ as large effects). The association between different dimensions of emotion processing, as well as the relationship to SSD pathology were expressed by Spearman's rank correlation coefficients. Fisher's $z$ tests were applied to test the difference of correlation strength between groups. Analyses were performed with IBM SPSS Version 22. To address the question whether alexithymia influences the association between emotion regulation and SSD pathology, a mediation analysis was planned. Furthermore, a mediation analysis was planned to investigate the effect of alexithymia and emotion regulation deficits on emotion recognition performance. However, for both mediation analyses, the variables did not match the requirements (i.e., did not correlate significantly).

\section{RESULTS}

\subsection{Group differences in emotion processing and emotion regulation}

For the TAS-20, a significant main effect of group $(F[3,66]=14.56$, $p<.001, \eta_{p}^{2}=.398$ ) was revealed (Figure 2). SSD patients had significantly higher alexithymia, as reflected in the TAS-20 total score ( $F[1$, $68]=28.95, p<.001, \eta_{p}{ }^{2}=.299$ ), as well as in the subscales DIF $\left(F[1,68]=41.93, p<.001, \eta_{p}{ }^{2}=.381\right)$ and $\operatorname{DDF}(F[1,68]=31.28$, $\left.p<.001, \eta_{p}{ }^{2}=.315\right)$ but not in the subscale EOT $(F[1,68]=1.50$, $p=.225, \eta_{p}{ }^{2}=.022$ ). 
For the Emotion Regulation Questionnaire, again a significant main effect of group was revealed $\left(F[2,67]=9.17, p<.001, \eta_{p}{ }^{2}=.215\right)$. SSD patients reported significantly less cognitive reappraisal ( $F[1$, $\left.68]=15.47, p<.001, \eta_{p}{ }^{2}=.185\right)$ but a trend towards more expressive suppression $\left(F[1,68]=3.36, p=.071, \eta_{p}^{2}=.047\right)$.

For emotion recognition abilities, we found a main effect of face valence $\left(F[2,67]=35.95, p<.001, \eta_{p}{ }^{2}=.518\right)$, as well as a face $x$ IAPS $\left(F[4,65]=3.62, p=.01, \eta_{p}{ }^{2}=.182\right)$ and a face $x$ group interaction $(F[2$, $\left.67]=3.86, p=.026, n_{p}^{2}=.103\right)$. Post-hoc $t$ tests revealed a statistical trend towards significance for patients with SSD recognizing more angry faces correctly (t[68] $=-1.686, p=.096, d=0.40$; Figure 4). Moreover, a trend for the IAPS category was revealed ( $F[2$, $67]=2.53, p=.087, \eta_{p}^{2}=.070$ ). The three-way interaction, however, was not significant. In addition, no main effect of group occurred.

Regarding the Trust Game, a Mann-Whitney $U$ test revealed significantly lower average investment rates in patients than in controls $(d=0.73)$. In addition, a significant main effect of group was revealed for the sympathy ratings $\left(F[1,47]=6.16, p=.017, \eta_{p}^{2}=.116\right)$ with patients giving lower ratings of sympathy than controls before $(d=0.85)$ and after $(d=0.53)$ the interaction. Furthermore, patients rated the trustees' behaviour as less fair than controls $(d=1.09$; see Table 2).

\subsection{Correlations between emotion regulation and the recognition of one's own versus other's emotions}

Exploratory correlation analyses revealed significant correlations between alexithymia subscales and emotion regulation strategies. Table 3 reports the correlations across all participants and additionally

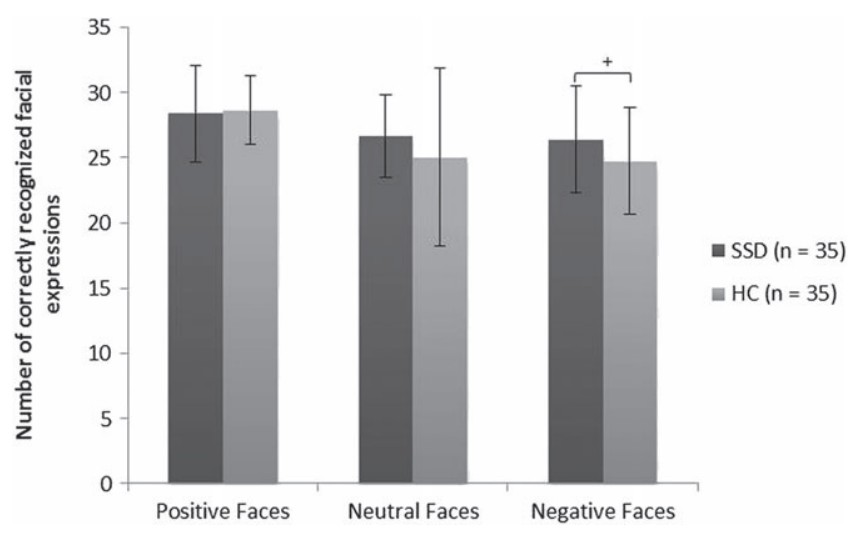

FIGURE 4 Number of correctly recognized positive, neutral, and negative facial expressions with standard deviations for patients (somatic symptom disorder [SSD]) and healthy controls (HC). ${ }^{+} p<.1$
TABLE 3 Correlations (Spearman's rho) between self-reported measures of alexithymia (TAS-20) and ERQ for the total sample (all; $n=70$ ) as well as for the HC $(n=35)$ and for the SSD $(n=35)$

\begin{tabular}{|c|c|c|c|c|c|c|}
\hline & & & & TAS-20 & & \\
\hline & & & DDF & DIF & EOT & Total \\
\hline \multirow[t]{6}{*}{ ERQ } & CR & $\mathrm{HC}$ & .071 & .178 & $-.366^{*}$ & -.163 \\
\hline & & SSD & -.181 & $-.383^{*}$ & -.241 & $-.372^{*}$ \\
\hline & & All & $-.301^{*}$ & $-.403^{* *}$ & $-.310^{* *}$ & $-.443^{* *}$ \\
\hline & ES & $\mathrm{HC}$ & $.546^{* *}$ & .158 & $.328^{+}$ & $.399^{*}$ \\
\hline & & SSD & $.581^{* *}$ & $.326^{+}$ & $.488^{* *}$ & $.531^{* *}$ \\
\hline & & All & $.556^{* *}$ & $.318^{* *}$ & $.424^{* *}$ & $.499^{* *}$ \\
\hline
\end{tabular}

Note. TAS-20 = Toronto Alexithymia Scale-20; DDF = difficulties describing feelings; DIF = difficulties identifying feelings; EOT = externally oriented thinking; Total = TAS-20 total score; ERQ = Emotion Regulation Questionnaire; $\mathrm{CR}=$ cognitive reappraisal; $\mathrm{ES}=$ expressive suppression; $\mathrm{HC}=$ healthy control group; SSD = somatic symptom disorder group. Significant correlations in bold print and significance levels are as follows.

${ }^{* *} p \leq .01$.

${ }^{*} p \leq .05$.

${ }^{+} p<.1$.

for both groups separately. Direct comparisons of the strength of correlations revealed that the correlation of cognitive reappraisal and DIF was significantly stronger in SSD than in healthy controls $(z=-2.33$, $p<.05)$.

There were no significant correlations between emotion regulation or alexithymia and the recognition of emotions in others but some statistical trends towards significance. A trend towards a positive correlation of the alexithymia subscale DDF and overall face recognition performance was observed $\left(r_{s}=.204, p=.09\right)$. Moreover, a trend towards a positive correlation of cognitive reappraisal and the recognition of negative faces was found in the control group $\left(r_{s}=.322\right.$, $p=.059)$. In the SSD group, we observed a trend towards a negative correlation of the recognition of negative faces and the TAS-20 total score $\left(r_{s}=-.301, p=.079\right)$. This correlation between the TAS-20 total score and the recognition of negative faces was significantly stronger in the SSD than in the healthy control group $(z=2.05, p<.05)$. It should be noted that in SSD, no significant correlation between emotion recognition and behaviour in the trust game was observed.

\subsection{Correlations between the processing and regulation of emotions and somatic symptoms}

Exploratory correlation analyses revealed significant positive correlations between both the number and the intensity of somatic symptoms and alexithymia subscales. Table 4 reports the correlations across all

TABLE 2 Trust Game scores $(M \pm S D)$ of patients (SSD; $n=18)$ and HC $(n=31)$ for average investment during the Trust Game, the sympathy ratings before and after the Trust Game and the ratings of the trustees fairness after the Trust Game

\begin{tabular}{llll} 
& $\mathbf{1}$ & $\mathbf{2}$ & $\begin{array}{l}\text { U test } \\
\mathbf{z} \text { value }\end{array}$ \\
\hline Average investment rate $(M \pm S D)$ & SSD & HC & $z=-2.425, p<.05$ \\
Sympathy rating before interaction $(M \pm S D)$ & $4.6 \pm 1.2$ & $5.6 \pm 1.6$ & $z=-2.831, p<.01$ \\
Sympathy rating after interaction $(M \pm S D)$ & $4.7 \pm 1.1$ & $5.5 \pm 1.0$ & $z=-2.031, p<.05$ \\
Fairness rating after interaction $(M \pm S D)$ & $4.8 \pm 1.1$ & $5.4 \pm 1.2$ & $z=-3.315, p<.001$ \\
\hline
\end{tabular}

Note. SSD = somatic symptom disorder group; $\mathrm{HC}=$ healthy control group; $M=$ mean; $S D=$ standard deviation. 
TABLE 4 Correlations (Spearman's rho) between self-reported measures of alexithymia (TAS-20) and ERQ and measures of somatic symptoms (SOMS-7T and PHQ-15) for the total sample (all; $n=70)$ as well as the HC $(n=35)$ and the SSD $(n=35)$

\begin{tabular}{|c|c|c|c|c|c|c|c|c|}
\hline & & & \multicolumn{3}{|l|}{ TAS-20 } & \multicolumn{3}{|c|}{ ERQ } \\
\hline & & & DDF & DIF & EOT & Total & CR & ES \\
\hline \multirow[t]{5}{*}{ SOMS } & Number of symptoms & $\mathrm{HC}$ & .213 & $.298^{+}$ & .023 & .174 & .221 & -.039 \\
\hline & & SSD & .110 & .249 & -.021 & .197 & -.173 & .081 \\
\hline & & All & $.432^{* * *}$ & $.577^{* * *}$ & .095 & $.446^{* * *}$ & $-.237^{*}$ & .144 \\
\hline & Intensity of symptoms & $\mathrm{HC}$ & .179 & .269 & -.038 & .119 & .257 & -.068 \\
\hline & & All & $.539^{* * *}$ & $.667^{* * *}$ & .121 & $.538^{* * *}$ & $-.335^{* *}$ & .187 \\
\hline \multirow[t]{3}{*}{ PHQ-15 } & & $\mathrm{HC}$ & .264 & $.620^{* * *}$ & $.303^{+}$ & $.416^{* *}$ & .014 & -.120 \\
\hline & & SSD & $.420^{*}$ & $.510^{* *}$ & $.287^{+}$ & $.546^{* *}$ & -.198 & .239 \\
\hline & & All & $.642^{* * *}$ & $.776^{* * *}$ & $.280^{*}$ & $.686^{* * *}$ & $-.421^{* * *}$ & $.217^{+}$ \\
\hline
\end{tabular}

Note. Tas-20 = Toronto Alexithymia Scale-20; DDF = difficulties describing feelings; DIF = difficulties identifying feelings; EOT = externally oriented thinking; Total = TAS-20 total score; ERQ = Emotion Regulation Questionnaire; $\mathrm{CR}=$ cognitive reappraisal; ES = expressive suppression; SOMS-7T = Screening for Somatoform Disorders; PHQ-15 = Patient Health Questionnaire; HC = healthy control group; SSD = somatic symptom disorder group. Significant correlations in bold print and significance levels are as follows.

$* * * \mathrm{p}<.001$.

${ }^{* *} p \leq .01$.

${ }^{*} p \leq .05$.

${ }^{+} p<.1$.

participants, as well as separately for the SSD and the healthy control group. None of the correlations between emotion processing and the measures of somatic symptoms differed significantly between groups (all $p$ values $\geq .4$ ).

\section{DISCUSSION}

SSD has been linked to impairments in emotion processing, which may contribute to the development and maintenance of medically unexplained physical complaints. Thus, emotion processing might be an important target for the psychotherapy of SSD. The aim of this study was to explore different aspects of emotion processing in SSD and to investigate how they might interact. To this end, we examined the understanding and the regulation of one's own feelings, the recognition of others' emotions and trust in patients with SSD, and a healthy control group.

We were able to replicate previous findings of deficits in the processing of one's own emotions in patients with SSD in comparison to healthy controls, indicated by higher levels of self-reported alexithymia (Bankier, Aigner, \& Bach, 2001; De Gucht \& Heiser, 2003; Pedrosa Gil et al., 2009). Our results are in line with earlier reports indicating that especially the identification and description of one's own feelings are affected in SSD (Deary et al., 1997). Consistently, recent studies highlight the particular importance of improving patients' abilities to understand their own feelings, and especially to identify them, when it comes to coping with bodily complaints (Bailer et al., 2017; Shibata et al., 2014).

In our sample, patients with SSD report using less cognitive reappraisal than healthy controls, indicating the insufficient application of cognitive strategies in order to regulate affective arousal in SSD, which is in agreement with previous studies (Bucci, 1997b; Taylor et al., 1997). According to our findings, patients with SSD tend to use expressive suppression more often than healthy controls to cope with negative feelings. Whereas cognitive reappraisal is believed to be more adaptive to handle negative feelings, suppression of emotion-expressive behaviour was found to increase the response of the sympathetic nervous system (Gross, 1998b; Gross, 2002). Hence, it is a strategy that is believed to further enhance physiological aspects of emotional experience and therefore promotes continued attention to bodily sensations (Alexander, 1950; Pennebaker \& Seagal, 1999; Traue, 1998).

Contrary to previous studies (Beck et al., 2013) and our hypotheses, our findings indicate that patients with SSD are as good as healthy controls in the recognition of others' emotions and even perform slightly better in the recognition of negative facial expressions. In contrast to the study of Buhlmann et al. (2006), we found no evidence for a negative bias in our emotion recognition task in patients with SSD. However, the better recognition rate of negative facial expressions might point to a higher sensitivity for negative emotions. It can be speculated that the higher sensitivity might be due to a sensitization caused by a higher incidence of negative reactions these patients experience in their social environment. These negative reactions might arise from persistent complaints about distressing symptoms and chronic help-seeking behaviour, which makes physicians and relatives feel increasingly strained and helpless (Kirmayer, Robbins, \& Paris, 1994).

Moreover, in our study, patients with SSD acted less trustful in the trust game, as they had lower average investment rates and lower sympathy and fairness ratings. In case this reduced trust would also occur in the medical context, it might contribute to a higher number of medical consultations and frequent changes of physicians, a well known pattern in SSD, so-called doctor hopping or doctor shopping (de Zwaan \& Müller, 2006). Although this is speculative, doctor hopping might arise not only from the frequent need of negative reinforcement but also from reduced trust in the physician's assurance of the patient's health. Moreover, related to the assumption of a higher sensitivity 
for negative emotions in others, SSD patients might be more sensitive to subtle signs of irritation in the doctor's face, making them doubt the doctor's trustworthiness.

Furthermore, our results show an association between the difficulties to understand own feelings and the application of maladaptive emotion regulation strategies: higher levels of alexithymia were related to a more frequent use of expressive suppression and less cognitive reappraisal. We found a trend-level association towards a better recognition of others' negative facial expressions through patients with lower TAS total scores and healthy controls who reported using cognitive reappraisal more often. These results are in line with previous studies that showed that the recognition of others' emotions is associated with the awareness of own feelings (Machado, Beutler, \& Greenberg, 1999). Although speculative, this shows that individuals capable of using their own emotions beneficially (i.e., are in touch with them as indicated by low TAS scores and constructively work on them as indicated by frequent cognitive reappraisal) also perform better in recognizing emotions in others.

There is evidence that high levels of alexithymia are linked to somatic amplification (Nakao \& Barsky, 2007; Wise \& Mann, 1994). Somatic amplification is supposed to aggravate patients' somatic complaints, as patients reinforce bodily sensations by keeping their attention shifted on the physiological aspects accompanying affective arousal (Barsky, Goodson, Lane, \& Cleary, 1988). Indeed, we found significant correlations between alexithymia and the amount and intensity of somatic symptoms, both across all participants and specifically in the SSD group. We also found a negative association between cognitive reappraisal and the number and intensity of somatic symptoms in the total sample. As mentioned above, in contrast to expressive suppression, this emotion regulation strategy is believed to be more adaptive and to have no detrimental effects on emotion-related bodily sensations (Gross, 1998a). Although we cannot draw causal conclusions from these correlations, they might suggest that alexithymia and the preferred emotion regulation strategy serve as predictors of the intensity of somatic symptoms, warranting a closer examination in future studies.

One of the main limitations of the study is the primarily assessment of different emotion regulation strategies by means of a questionnaire that, first, only measures two ways of coping with negative feelings, and second, like self-report questionnaires in general, might underlie inaccuracy of recall (Coughlin, 1990). In addition, we did not assess current negative affect that might be the link between sensitization and negative emotions of others, reduced trust, and bodily symptoms. Future studies should apply additional experimental paradigms combined with psychophysiological measurement of mood induction, to take a closer look at the dissociation of understanding one's own emotions and the physical perception of accompanying physiological aspects. These studies could also experimentally investigate emotion regulation in SSD, possibly including additional emotion regulation strategies, such as acceptance. In our experimental design to assess emotion recognition, we combined the presentation of emotional scenes with facial expressions. The assumption was that negative scenes would require emotion regulation for correct emotion recognition. However, we found neither a systematic effect of the valence of the scenes on emotion recognition nor an interaction with group. Thus, we suggest that there is no direct link between emotion dysregulation and emotion recognition in SSD (which is also supported by a lack of significant correlations between emotion recognition performance and emotion regulation as reported in the questionnaires in the SSD group). Another limitation relates to the very brief assessment of trust, which should be investigated more systematically by applying more sophisticated experimental paradigms in future studies. Further, not all of our participants attended the trust game, which is why the sample for analysing this paradigm was small and the generalizability of these results can be questioned. Future studies should use larger sample sizes to investigate emotion processing in SSD, in particular replicating our findings of reduced trust in SSD. Furthermore, regarding the assumption that less trust might be linked to "doctor hopping," it would be of highest interest to assess the frequency of medical consultations in this context as well.

Our results replicate earlier findings of higher alexithymia and deficits in emotion regulation in patients diagnosed with previous versions of the DSM in a sample of patients with SSD and point to the important link between understanding one's own feelings and adaptive emotion regulation. We could not replicate the finding that patients with SSD have difficulties identifying others' emotions but on the contrary found slightly improved emotion recognition skills. Thus, our results indicate a dissociation in the emotion processing abilities of SSD patients, with impairments in understanding and regulating one's own emotions but an intact perception of others' feelings. These impairments might promote intolerance towards one's own negative feelings and their accompanying physiological sensations, whereas a sensitization towards others' negative emotions and being less trustful in social interactions might make negative feelings even more likely to occur. We assume that these factors may constitute a vicious circle contributing to the maintenance of medically unexplained somatic symptoms (Figure 5): If patients with SSD lack trust in others, this might lead to a higher incidence of negative feelings in social interactions, which

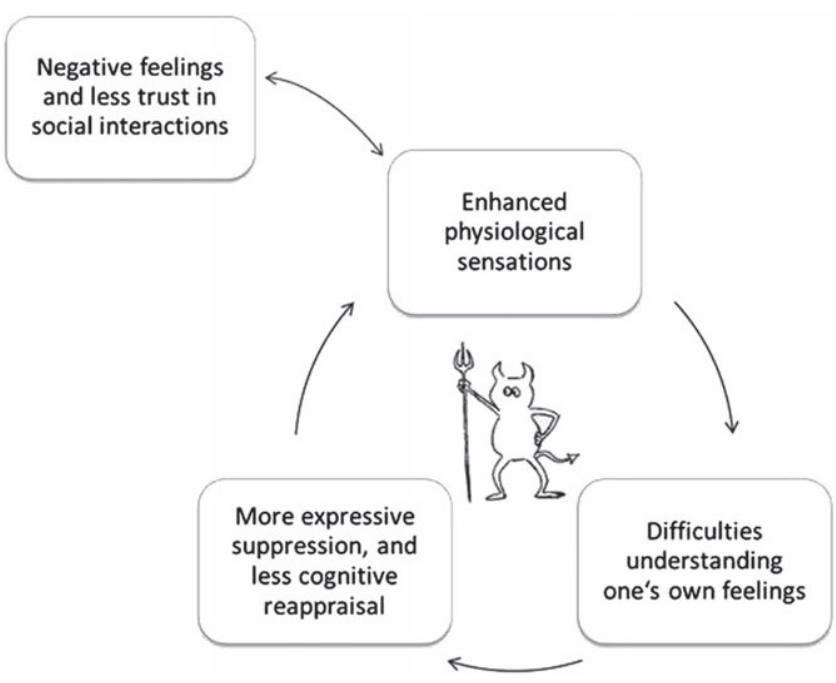

FIGURE 5 Vicious circle illustrating the possible influence of emotion processing impairments on the maintenance of medically unexplained somatic symptoms. Since feelings are accompanied by bodily sensations, the difficulties in understanding and regulating feelings may contribute to the misinterpretation and enhancement of physiological sensations and result in persisting somatic complaints 
they have difficulties to understand. In addition, accompanying bodily sensations might arise, which the patients fail to link to preceding emotional experiences. Due to their limited abilities to use cognitive strategies, they might intuitively rely more on expressive suppression in order to regulate affective arousal, which then will further enhance physiological sensations. Interpreting those sensations as signs of disease will most probably lead to further negative feelings and so forth.

The pattern of reduced trust behaviour and higher sensitivity to negative facial expressions in SSD is a new finding that might inform cognitive-behavioural models of SSD and will hopefully stimulate new research on social perception in SSD. A direct implication of our study for psychotherapy is that beyond emotion regulation training itself, improving the understanding of one's own emotions might present a crucial first step in the treatment of emotion processing deficits in SSD.

\section{ORCID}

Peter Kirsch (1) http://orcid.org/0000-0002-0817-1248

Daniela Mier (10) http://orcid.org/0000-0003-2518-7492

\section{REFERENCES}

Abler, B., \& Kessler, H. (2009). Emotion regulation questionnaire-Eine deutschsprachige Fassung des ERQ von Gross und John. Diagnostica, 55(3), 144-152.

Alexander, F. (1950). Psychosomatic medicine. Its principles and applications. New York: WW Norton \& Co. Press Google Scholar.

American Psychiatric Association (2013). Diagnostic and statistical manual of mental disorders (5th ed.). Arlington: American Psychiatric Publishing.

Bagby, R. M., Parker, J. D., \& Taylor, G. J. (1994). The twenty-item Toronto Alexithymia Scale-I. Item selection and cross-validation of the factor structure. Journal of Psychosomatic Research, 38(1), 23-32.

Bailer, J., Witthöft, M., Erkic, M., \& Mier, D. (2017). Emotion dysregulation in hypochondriasis and depression. Clinical Psychology \& Psychotherapy.

Bankier, B., Aigner, M., \& Bach, M. (2001). Alexithymia in DSM-IV disorder: Comparative evaluation of somatoform disorder, panic disorder, obsessive-compulsive disorder, and depression. Psychosomatics, 42(3), 235-240. https://doi.org/10.1176/appi.psy.42.3.235

Barsky, A. J., Goodson, J. D., Lane, R. S., \& Cleary, P. D. (1988). The amplification of somatic symptoms. Psychosomatic Medicine, 50(5), 510-519.

Beck, T., Breuss, M., Kumnig, M., \& Schussler, G. (2013). The first step is the hardest-Emotion recognition in patients with somatoform disorders. Zeitschrift für Psychosomatische Medizin und Psychotherapie, 59(4), 385-390.

Bucci, W. (1997a). Symptoms and symbols: A multiple code theory of somatization. Psychoanalytic Inquiry, 17(2), 151-172.

Bucci, W. (1997b). Symptoms and symbols: A multiple code theory of somatization. Psychoanalytic Inquiry: A Topical Journal for Mental Health Professionals, 17(2), 151-172. https://doi.org/10.1080/ 07351699709534117

Buhlmann, U., Etcoff, N. L., \& Wilhelm, S. (2006). Emotion recognition bias for contempt and anger in body dysmorphic disorder. Journal of Psychiatric Research, 40(2), 105-111. https://doi.org/10.1016/j. jpsychires.2005.03.006

Burton, C., Weller, D., \& Sharpe, M. (2009). Functional somatic symptoms and psychological states: An electronic diary study. Psychosomatic Medicine, 71(1), 77-83. https://doi.org/10.1097/PSY.0b013e31818f2acb

Coughlin, S. S. (1990). Recall bias in epidemiologic studies. Journal of Clinical Epidemiology, 43(1), 87-91.
Deary, I. J., Scott, S., \& Wilson, J. A. (1997). Neuroticism, alexithymia and medically unexplained symptoms. Personality and Individual Differences, 22(4), 551-564.

De Gucht, V., \& Heiser, W. (2003). Alexithymia and somatisation: Quantitative review of the literature. Journal of Psychosomatic Research, 54(5), 425-434.

de Zwaan, M., \& Müller, A. (2006). Doctor shopping: The difficult-tomanage patient. Wiener Medizinische Wochenschrift (1946), 156(15-16), 431-434.

Dillmann, U., Nilges, P., Saile, H., \& Gerbershagen, H. (1994). Assessing disability in chronic pain patients. Schmerz (Berlin, Germany), 8(2), 100-110.

Fenske, S., Lis, S., Liebke, L., Niedtfeld, I., Kirsch, P., \& Mier, D. (2015). Emotion recognition in borderline personality disorder: Effects of emotional information on negative bias. Borderline Personal Disord Emot Dysregul, 2, 10. https://doi.org/10.1186/s40479-015-0031-z

Franzen, N., Hagenhoff, M., Baer, N., Schmidt, A., Mier, D., Sammer, G., ... Lis, S. (2011). Superior "theory of mind"in borderline personality disorder: An analysis of interaction behavior in a virtual trust game. Psychiatry Research, 187(1), 224-233.

Gottschalk, J.-M., Bleichhardt, G., Kleinstäuber, M., Berking, M., \& Rief, W. (2015). Erweiterung der kognitiven verhaltenstherapie um emotionsregulationstraining bei patienten mit multiplen somatoformen symptomen: Ergebnisse einer kontrollierten pilotstudie. Verhaltenstherapie, 25(1), 13-21.

Gottschalk, J. M., \& Rief, W. (2012). Psychotherapeutische ansätze für patienten mit somatoformen störungen. Nervenarzt, 83(9), 1115-1127. https://doi.org/10.1007/s00115-011-3445-x

Gross, J. J. (1998a). Antecedent- and response-focused emotion regulation: Divergent consequences for experience, expression, and physiology. Journal of Personality and Social Psychology, 74(1), 224-237.

Gross, J. J. (1998b). The emerging field of emotion regulation: An integrative review. Review of General Psychology, 2(3), 271.

Gross, J. J. (2002). Emotion regulation: Affective, cognitive, and social consequences. Psychophysiology, 39(3), 281-291. https://doi.org/10.1017. S0048577201393198

Haller, H., Cramer, H., Lauche, R., \& Dobos, G. (2015). Somatoform disorders and medically unexplained symptoms in primary care. Deutsches Ärzteblatt International, 112(16), 279-287. https://doi.org/10.3238/ arztebl.2015.0279

Hanssen, D. J., Lucassen, P. L., Hilderink, P. H., Naarding, P., \& Voshaar, R. C. O. (2016). Health-related quality of life in older persons with medically unexplained symptoms. The American Journal of Geriatric Psychiatry, 24(11), 1117-1127.

Hautzinger, M., Keller, F., \& Kühner, C. (2006). Beck depressions-inventar: BDI II. RevisionHarcourt Test Services Frankfurt/Main.

Kessler, H., Kammerer, M., Hoffmann, H., \& Traue, H. C. (2010). Regulation von emotionen und alexithymie: Eine korrelative studie. PPmPPsychotherapie. Psychosomatik· Medizinische Psychologie, 60(05), 169-174.

Kirmayer, L. J., Robbins, J. M., \& Paris, J. (1994). Somatoform disorders: Personality and the social matrix of somatic distress. Journal of Abnormal Psychology, 103(1), 125.

Kleinstäuber, M., Gottschalk, J., Berking, M., Rau, J., \& Rief, W. (2016). Enriching cognitive behavior therapy with emotion regulation training for patients with multiple medically unexplained symptoms (ENCERT): Design and implementation of a multicenter, randomized, activecontrolled trial. Contemporary Clinical Trials, 47, 54-63.

Kroenke, K., Spitzer, R. L., \& Williams, J. B. (2002). The PHQ-15: Validity of a new measure for evaluating the severity of somatic symptoms. Psychosomatic Medicine, 64(2), 258-266.

Laloyaux, J., Fantini, C., Lemaire, M., Luminet, O., \& Laroi, F. (2015). Evidence of contrasting patterns for suppression and reappraisal emotion regulation strategies in alexithymia. The Journal of Nervous and Mental Disease, 203(9), 709-717. https://doi.org/10.1097/ NMD.0000000000000353 
Lang, P. J., Bradley, M. M., \& Cuthbert, B. N. (1999). International affective picture system (IAPS): Instruction manual and affective ratings. In The center for research in psychophysiologyUniversity of Florida.

Leonidou, C., Panayiotou, G., Bati, A., \& Karekla, M. (2016). Coping with psychosomatic symptoms: The buffering role of psychological flexibility and impact on quality of life. Journal of Health Psychology. 1359105316666657

Lis, S., Schönwetter, T., Mier, D., Gallhofer, B., \& Kirsch, P. (2011). Gestörte integration sozial-emotionaler Hinweisreize bei schizophrenen Patienten. Nervenheilkunde, 30(6), 385-393.

Lumley, M. A., Stettner, L., \& Wehmer, F. (1996). How are alexithymia and physical illness linked? A review and critique of pathways. Journal of Psychosomatic Research, 41(6), 505-518.

Machado, P. P., Beutler, L. E., \& Greenberg, L. S. (1999). Emotion recognition in psychotherapy: Impact of therapist level of experience and emotional awareness. Journal of Clinical Psychology, 55(1), 39-57.

MacLean, P. D. (1949). Psychosomatic disease and the "visceral brain": Recent developments bearing on the Papez theory of emotion. Psychosomatic Medicine, 11(6), 338-353.

Matzke, B., Herpertz, S. C., Berger, C., Fleischer, M., \& Domes, G. (2014). Facial reactions during emotion recognition in borderline personality disorder: A facial electromyography study. Psychopathology, 47(2), 101-110. https://doi.org/10.1159/000351122

Mewes, R., Rief, W., Stenzel, N., Glaesmer, H., Martin, A., \& Brahler, E. (2009). What is "normal" disability? An investigation of disability in the general population. Pain, 142(1-2), 36-41. https://doi.org/ 10.1016/j.pain.2008.11.007

Nakao, M., \& Barsky, A. J. (2007). Clinical application of somatosensory amplification in psychosomatic medicine. Biopsychosoc Med, 1, 17. https://doi.org/10.1186/1751-0759-1-17

Nemiah, J. C., \& Sifneos, P. E. (1970). Affect and fantasy in patients with psychosomatic disorders. Modern Trends in Psychosomatic Medicine, 2, 26-34.

Pedrosa Gil, F., Ridout, N., Kessler, H., Neuffer, M., Schoechlin, C., Traue, H. C., \& Nickel, M. (2009). Facial emotion recognition and alexithymia in adults with somatoform disorders. Depression and Anxiety, 26(1), E26-E33. https://doi.org/10.1002/da.20456

Pennebaker, J. W., \& Seagal, J. D. (1999). Forming a story: The health benefits of narrative. Journal of Clinical Psychology, 55(10), 1243-1254. https://doi.org/10.1002/(SICI)1097-4679(199910)55:10<1243::AIDJCLP6>3.0.CO;2-N

Rask, M. T., Ørnbøl, E., Rosendal, M., \& Fink, P. (2017). Long-term outcome of bodily distress syndrome in primary care: A follow-up study on health care costs, work disability, and self-rated health. Psychosomatic Medicine, 79(3), 345-357.

Rief, W., \& Hiller, W. (2008). Screening für somatoforme störungen (SOMS) Hogrefe.

Rief, W., \& Martin, A. (2014). How to use the new DSM-5 somatic symptom disorder diagnosis in research and practice: A critical evaluation and a proposal for modifications. Annual Review of Clinical Psychology, 10, 339-367. https://doi.org/10.1146/annurev-clinpsy032813-153745

Saß, H., Wittchen, H.-U., \& Zaudig, M. (1996). Diagnostisches und statistisches manual psychischer störungen-DSM-IV. Deutsche bearbeitung $u$. Einleitung von H. Sass, H.-U. Wittchen, M. Zaudig.

Sauer, N., \& Eich, W. (2007). Somatoforme störungen und funktionsstörungen. Dtsch Arztebl, 104(1-2), 45-53.

Schönenberg, M., Mares, L., Smolka, R., Jusyte, A., Zipfel, S., \& Hautzinger, M. (2014). Facial affect perception and mentalizing abilities in female patients with persistent somatoform pain disorder. European Journal of Pain, 18(7), 949-956. https://doi.org/10.1002/j.1532-2149.2013.00440.x
Shibata, M., Ninomiya, T., Jensen, M. P., Anno, K., Yonemoto, K., Makino, S., ... Imada, Y. (2014). Alexithymia is associated with greater risk of chronic pain and negative affect and with lower life satisfaction in a general population: The Hisayama study. Plos One, 9(3), e90984.

Spitzer, R. L., Kroenke, K., Linzer, M., Hahn, S. R., Williams, J. B., Verloin de Gruy, F., ... Davies, M. (1995). Health-related quality of life in primary care patients with mental disorders: Results from the PRIME-MD 1000 study. JAMA, 274(19), 1511-1517.

Subic-Wrana, C., Beutel, M. E., Knebel, A., \& Lane, R. D. (2010). Theory of mind and emotional awareness deficits in patients with somatoform disorders. Psychosomatic Medicine, 72(4), 404-411. https://doi.org/ 10.1097/PSY.Ob013e3181d35e83

Swart, M., Kortekaas, R., \& Aleman, A. (2009). Dealing with feelings: Characterization of trait alexithymia on emotion regulation strategies and cognitive-emotional processing. Plos One, 4(6), e5751. https://doi.org/ 10.1371/journal.pone.0005751

Taylor, G. J. (2000). Recent developments in alexithymia theory and research. Canadian Journal of Psychiatry, 45(2), 134-142.

Taylor, G. J., Bagby, R. M., \& Parker, J. D. A. (1997). Disorders of affect regulation: Alexithymia in medical and psychiatric illness. Cambridge, England: Cambridge University Press. 10.1017. СBO9780511526831.

de Timary, P., Roy, E., Luminet, O., Fillee, C., \& Mikolajczak, M. (2008). Relationship between alexithymia, alexithymia factors and salivary cortisol in men exposed to a social stress test. Psychoneuroendocrinology, 33(8), 1160-1164. https://doi.org/10.1016/j.psyneuen.2008.06.005

Tottenham, N., Tanaka, J. W., Leon, A. C., McCarry, T., Nurse, M., Hare, T. A., ... Nelson, C. (2009). The NimStim set of facial expressions: Judgments from untrained research participants. Psychiatry Research, 168(3), 242-249. https://doi.org/10.1016/j.psychres.2008.05.006

Traue, H. C. (1998). Emotion und hesundheit: Die psychobiologische regulation durch hemmungenSpektrum, Akad. Verlag.

Uher, T. (2010). Alexithymia and immune dysregulation: A critical review. Activitas Nervosa Superior, 52(1), 40-44.

Waller, E., \& Scheidt, C. E. (2004). Somatoform disorders as disorders of affect regulation: A study comparing the TAS-20 with non-self-report measures of alexithymia. Journal of Psychosomatic Research, 57(3), 239-247. https://doi.org/10.1016/S0022-3999(03)00613-5

Waller, E., \& Scheidt, C. E. (2006). Somatoform disorders as disorders of affect regulation: A development perspective. International Review of Psychiatry, 18(1), 13-24. https://doi.org/10.1080/ 09540260500466774

Wise, T. N., \& Mann, L. S. (1994). The relationship between somatosensory amplification, alexithymia, and neuroticism. Journal of Psychosomatic Research, 38(6), 515-521.

Wittchen, H.-U., \& Jacobi, F. (2001). Die versorgungssituation psychischer störungen in Deutschland eine klinisch-epidemiologische abschätzung anhand des bundes-gesundheitssurveys 1998. Bundesgesundheitsblatt, Gesundheitsforschung, Gesundheitsschutz, 44(10), 993-1000.

Wittchen, H.-U., Zaudig, M., \& Fydrich, T. (1997). SKID. In Strukturiertes clinisches interview für DSM-IV. Achse I und II. Göttingen: Hogrefe.

Zaki, J., \& Williams, W. C. (2013). Interpersonal emotion regulation. Emotion, 13(5), 803. 